\title{
APPLICATION OF THE SPIN GLASS FORMALISM TO THE QUADRUPOLAR GLASS $\mathrm{Na}(\mathrm{CN})_{x} \mathrm{Cl}_{1-x}$
}

\author{
K. WALASEK \\ Institute of Physics, Pedagogical University of Zielona Góra \\ Pl. Słowiański 6, 65-069 Zielona Góra, Poland
}

\begin{abstract}
A new model of quadrupolar glass of mixed alkali halide-cyanide crystals is proposed. This model is treated by the Sherrington-Kirkpatrick like procedure. Physical consequences of our approach are discussed. The temperature dependence of the quadrupolar Edwards-Anderson order parameter and elastic constant $C_{44}$ near the quadrupolar glass transition was obtained.
\end{abstract}

PACS numbers: $64.60 . \mathrm{Cn}, 64.70 . \mathrm{Pf}, 62.20 . \mathrm{Dc}, 75.10 . \mathrm{Nr}$

The mixed alkali halide-cyanide crystals such as $\mathrm{Na}(\mathrm{CN})_{x} \mathrm{Cl}_{1-x}$, $\mathrm{K}(\mathrm{CN})_{x} \mathrm{Br}_{1-x}$ etc. with $x<x_{\mathrm{c}}$ form below some temperature $T_{\mathrm{g}}(x)$ a special phase, which is called an orientational or quadrupolar glass $(\mathrm{QG})$ (cf. e.g. the review of Binder and Reger [1]). At $T>T_{\mathrm{g}}(x)$ these systems show the cubic structure, where the sides of anions are statistically occupied by halide ions and dumbbell-shaped $\mathrm{CN}^{-}$molecular groups, which rotate nearly freely. When the temperature is lowered and crosses $T_{g}(x), \mathrm{CN}^{-}$ions freeze into random directions. To the lowest temperature no long range order is observed and the global symmetry of the system remains cubic. This can be compared with the random freeze-out of the spins in a canonical spin glass. However, the frustration which leads to the QG ordering is more subtle and more complex than in spin systems. In addition, because of the coupling between rotational and translational degrees of freedom [2] the random freezing of orientations of $\mathrm{CN}^{-}$ions leads to local static lattice deformations and hence QG has some properties of a structural glass.

Various experiments provide an evidence for an existence of QG phase of mixed alkali halide-cyanide crystals [3]. One of basic characteristic is the temperature dependence of the central peak intensity of quasi-elastic neutron scattering [3] and of the NMR frequency distribution [4]. An important feature of QG transition is the considerable softening and successive hardening of the elastic constant $C_{44}$ [3].

In view of the limited space available for the present work, we will restrict ourselves to a short presentation of the proposed model and its physical consequences. A detailed description is given elsewhere [5].

The starting point is the semi-microscopic Hamiltonian obtained in a result of a coarse-graining procedure [6]. It contains the following effective interactions: 
1. The potential energy of the lattice, which is treated as an elastic medium. This part of the Hamiltonian is quadratic in the local strain tensor.

2. The coupling of the defects $\left(\mathrm{CN}^{-}\right.$ions) to the displacement field written as a bilinear form of the local strain tensor and local stress field [6].

3. The anisotropy potential dependent of the orientation of a defect.

4. The effective orientational coupling between defects, containing contributions from direct multipole interactions as well as indirect interactions via lattice distortions on the short length scale, which has been eliminated during the coarse-graining procedure. This coupling is considered as long ranged [6].

The quenched site disorder of $\mathrm{CN}^{-}$at the microscopic level [2] leads to the random orientational coupling, anisotropy potential and local stress field. In contrast to the approach of Ref. [6], where $\mathrm{CN}^{-}$ions have a limited number of orientational states we impose no limitations of $\mathrm{CN}^{-}$ion treating it, similarly as in the microscopic Michel theory [2], as a quadrupolar molecular group.

Due to the form of the above discussed Hamiltonian in the calculation of the partition function the integration over strain fluctuations can be performed exactly as a sequence of Gaussian integrals. As a result, one obtains the Hamiltonian containing only orientational interactions, which reads

$$
H_{\mathrm{orient}}=-\sum_{\boldsymbol{x}} \sum_{\lambda=1}^{5} h_{\lambda}(\boldsymbol{x}) Y_{\lambda}\left(\Omega_{\boldsymbol{x}}\right)-\frac{1}{2} \sum_{\boldsymbol{x} \neq \boldsymbol{y}} \sum_{\lambda, \lambda^{\prime}=1}^{5} u_{\lambda \lambda^{\prime}}(\boldsymbol{x}, \boldsymbol{y}) Y_{\lambda}\left(\Omega_{\boldsymbol{x}}\right) Y_{\lambda^{\prime}}\left(\Omega_{\boldsymbol{y}}\right),(1)
$$

where $Y_{\lambda}\left(\Omega_{x}\right),(\lambda=1 \ldots 5)$ denotes the symmetry-adapted spherical harmonic [2] related to the $\lambda$-th component of the quadrupolar momentum of $\mathrm{CN}^{-}$ion located at the site $x$ of the coarse-grained lattice (the small dipolar momentum can be neglected [2]), $\Omega_{x}$ denotes a pair of angles specifying an orientation of the defect, and $h_{\lambda}(x)$ and $u_{\lambda \lambda^{\prime}}(x, y)$ are, respectively, the random strains field and long ranged orientational coupling.

In order to obtain the free energy averaged over the quenched disorder we use the replica trick and Sherrington-Kirkpatrick-like approach. Within the replica symmetric theory the free energy is a function $q_{\mathrm{EA}}+\bar{\Delta}^{2}$, where $q_{\mathrm{EA}}$ denotes the QG Edwards-Anderson parameter and $\bar{\Delta}=\Delta / J$. The squared parameters $\Delta$ and $J$ are proportional to the variances of the quenched random quantities $h_{\lambda}(\boldsymbol{x})$ and $u_{\lambda \lambda^{\prime}}(x, y)$, respectively and $q_{\mathrm{EA}}$ is defined as follows [4]:

$$
q_{\mathrm{EA}}=\sum_{\lambda}\left[\left\langle Y_{\lambda}\left(\Omega_{\boldsymbol{x}}\right)\right\rangle_{T}^{2}\right]_{\mathrm{av}},
$$

where $\langle\ldots\rangle$ and $[\ldots]_{a v}$ denote, respectively, the thermal and sample averages.

In QG phase there is no long-range order, therefore the components of the global quadrupolar momentum $\left[\left\langle Y_{\lambda}\left(\Omega_{\boldsymbol{x}}\right)\right\rangle\right]_{\mathrm{av}}$ vanish. The local ordering of $\mathrm{CN}^{-}$ ions is characterized by $\left[\left\langle Y_{\lambda}\left(\Omega_{x}\right)\right\rangle^{2}\right]_{\mathrm{av}}$. The parameter $q_{\mathrm{EA}}(1 \mathrm{a})$ plays a similar role of the Edwards-Anderson parameter in spin systems. A convenient way to measure $q_{\mathrm{EA}}(2)$ is the quasi-elastic neutron scattering and NMR experiment because $q_{\mathrm{EA}}$ is proportional to the central peak intensity $[2,3]$ and second moment of NMR spectrum [3, 4]. In our semi-microscopic approach $\Delta$ and $J$ cannot be calculated from the first principles and are treated as adjustable parameters (cf. e.g. Ref. [4]). 
The elastic constant $C_{44}$ has the following form [5]:

$$
C_{44}=C_{44}^{0}\left[1+\frac{B^{2}}{20 \pi J C_{44}^{0}} \chi_{\mathrm{QG}}\right]^{-1} \text {, }
$$

where $C_{44}^{0}$ is the bare elastic constant, the parameter $B$ measures the strength of the coupling of the orientational modes of $T_{2 g}$ symmetry to the displacement field [2] and $\chi_{\mathrm{QG}}=\left(1-4 \pi q_{\mathrm{EA}} / 5\right) / \bar{T}$ with $\bar{T}=T / J$ is the orientational susceptibility.

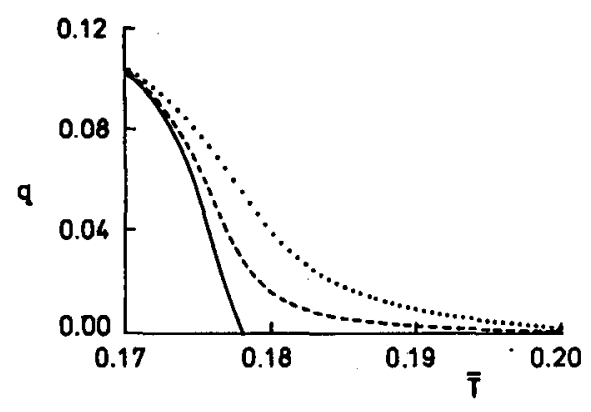

Fig. 1. The temperature dependence of quadrupolar glass Edwards-Anderson parameter for $\bar{\Delta}=0$ (solid line), $\bar{\Delta}=0.05$ (dashed line) and $\bar{\Delta}=0.1$ (*). Here $\bar{T}$ denotes $k_{\mathrm{B}} T$ scaled by the parameter $J$.

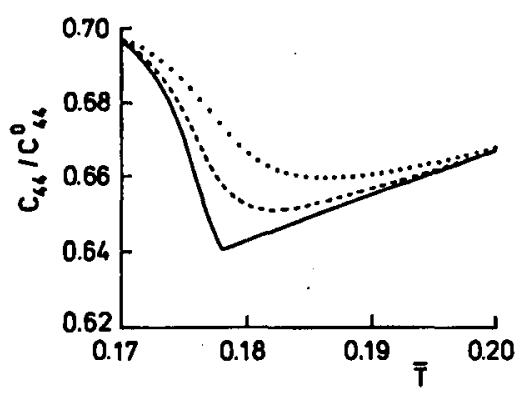

Fig. 2. The temperature dependence of the elastic constant $C_{44}$ scaled by the bare one $C_{44}^{0}$ for $\bar{\Delta}=0$ (solid line), $\bar{\Delta}=0.05$ (dashed line) and for $\bar{\Delta}=0.1(*)$. Here $\bar{T}$ denotes $k_{\mathrm{B}} T$ measured in the units of the parameter $J$.

In Fig. 1 the temperature dependence ( $T$-dependence) of $q_{\mathrm{EA}}$ obtained within our theory for $\bar{\Delta}=0,0.05$ and 0.1 is shown. It is seen that in the presence of the random strains field the sharp continuous QG transition is smeared out similarly as in spin or proton glasses [7]. The variation of $q_{\mathrm{EA}}$ with the temperature shown in Fig. 1 is very similar to the $T$-dependence of the second moment of the ${ }^{23} \mathrm{Na}$ satellite distribution measured in $\mathrm{Na}(\mathrm{CN})_{x} \mathrm{Cl}_{1-x}$ system [4] and of the elastic diffuse intensity neutron scattering [3]. 
In Fig. 2 the $T$-dependence of $C_{44} / C_{44}^{0}$ is presented. We have assumed that $B^{2} /\left(20 \pi J C_{44}^{0}\right)=0.1$. In the presence of the random strain field the sharp minimum, which appears at $\bar{T}_{\mathrm{g}}$ for $\bar{\Delta}=0$ is flattened. This effect becomes more apparent with an increase in $\bar{\Delta}$. The form of $T$-dependence of $C_{44}$ agrees with that measured experimentally [3].

Summarizing, the phenomenon of ordering of $\mathrm{CN}^{-}$molecular groups in mixed alkali halide-cyanide crystals can be studied using the methods developed in the theory of canonical spin glasses. The obtained results seem to be reasonable.

\section{References}

[1] K. Binder, J. Reger, Adv. Phys. 41, 547 (1992).

[2] K.H. Michel, J. M. Rowe, Phys. Rev. B 22, 1417 (1980); K.H. Michel, Phys. Rev. $B$ 35, 1405 (1987).

[3] U.T. Höchli, K. Knorr, A. Loidl, Adv. Phys. 39, 405 (1990).

[4] W. Wiotte, J. Peterson, R. Blinc, S. Elschner, Phys. Rev. B 43, 12751 (1991).

[5] K. Walasek, K. Lukierska-Walasek, unpublished.

[6] H. Vollmayr, R. Kree, A. Zippelius, Phys. Rev. B 44, 12238 (1991).

[7] R. Pirc, B. Tadić, R. Blinc, Phys. Rev. B 36, 8607 (1987). 